\section{Equilibrium Diagram for the Nickel-Cadmium System}

'To study the effect of size factor in alloying behaviour it was decided to determine the ternary system $\mathrm{Cu}-\mathrm{Ni}-\mathrm{Cd}$, on which no data have been published. The binary systems $\mathrm{Cu}-\mathrm{Ni}$ and $\mathrm{Cu}-\mathrm{Cd}$ have been fully investigated, the results of the various investigations being summarized by Hansen ${ }^{1}$. Of the Ni-Cd system only cadmium-rich alloys have been studied in detail a further investigation of this system was therefore carried out in conjunction with the work on the ternary diagram.

Alloys were prepared from analytical reagent quality nickel powder and stick cadmium; the powder was given a reducing treatment in hydrogen to remove any oxide present. Weighed amounts of the pure metals were sealed in evacuated silica capsules for melting, which was carried out by heating for about $1 \mathrm{~h}$ at $1,200^{\circ}-1,250^{\circ} \mathrm{C}$, conditions which had been shown to be adequate for the nickel to be taken into solution. The capsules were then rapidly cooled to give a fine-grained ingot and annealed for a minimum period of three weeks to homogenize the alloy. At the end of the annealing period the alloys were water-quenched and then weighed to check whether any loss had occurred in melting. The nominal compositions of ingots which were satisfactory in this respect were taken as the actual compositions.

The liquidus surface for the $\mathrm{Ni}-\mathrm{Cd}$ systems was examined by equilibrating nickel in the form of wire with cadmium at constant temperature. At the end of the soaking period the capsulo containing the sample was quenched and broken open. The residuum of the wire was separated from the melt and the latter analysed for its nickel content. The results obtained from this study are indicated in Fig. 1. Cooling curves on alloys close to 50 atom per cent nickel failed to show any arrest between the liquidus and $502^{\circ} \mathrm{C}$; micro-examination of these cooling curve ingots showed the formation of peritectic walls of $\beta \mathrm{Ni}-\mathrm{Cd}$ surrounding dendrites of the $\alpha$-phase. The peritectic temperature was therefore determined from heating curves on homogenized alloys and gave a value of $695^{\circ} \mathrm{C}$ for the decomposition temperature of the $B$ phase. The formation of the $\gamma^{\prime}$ phase was studied by combined dilatometry and thermal analysis. The volume change at $405^{\circ}$ reported by Voss ${ }^{2}$ was not observed; an alloy of $\gamma^{\prime}$ together with a small amount of $\gamma$ showed an arrest point accompanied by partial collapse of the specimen at $490^{\circ} \mathrm{C}$ and a second arrest and complete collapse at $502^{\circ} \mathrm{C}$. It is significant that Voss, who reported a peritectic temperature of $502^{\circ}$, obtained this value on higher nickel content alloys ( $\sim 10$ wt. per cent nickel) than Swartz and Phillips ${ }^{3}$ (6-8 wt. per cent nickel), and it is probable the former detected the peritectic involving $\gamma^{\prime}$ and the latter that of $\gamma$, which would be more prominent in the lower nickel alloys

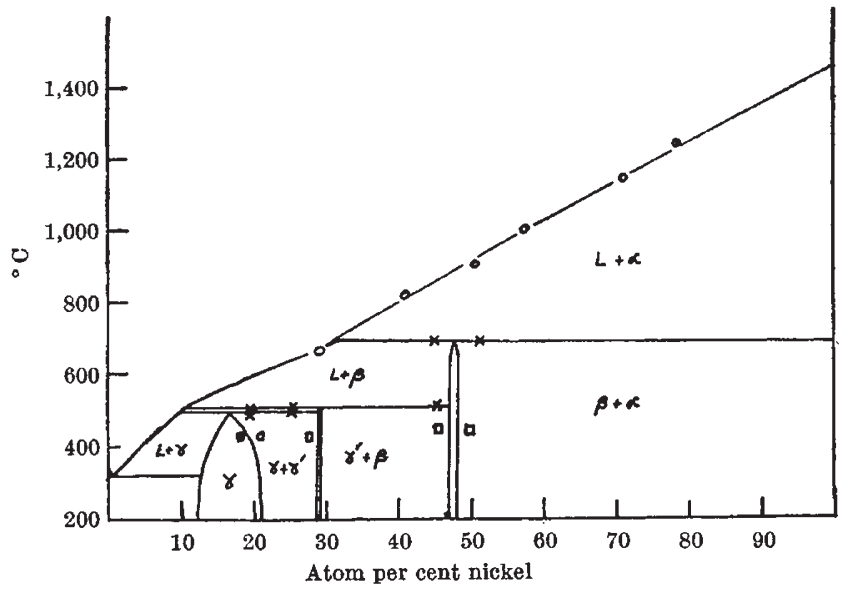

Fig. 1. $\times$, Thermal analysis. $O$, equilibration; $\square$, single phase; they studied. The nickel-rich solubility limit is confirmed by an alloy of 21.2 atom per cent nickel, which showed the presence of a trace amount of $\gamma^{\prime}$ at $430^{\circ} \mathrm{C}$ compared to a reported solubility of 19.5 atom per cent at this tem. perature. Other than the $\beta$-phase (Ni-Cd) no other compounds were observed in the system.

The $\beta$-phase structure has been studied by X-ray diffraction using the powder method. The compound possesses the structure $F d 3 m$ with a cell edge of $11.40 \mathrm{kX}$ units and contains approximately 48 formula units in the unit cell.

R. J. LOTT

Department of Chemistry,

J. K. CRitchuey

Brunel College,

London, W.3.

${ }^{1}$ Hansen, M., Constitution of Binary Alloys (McGraw-Hill, 1958).

${ }^{2}$ Voss, G., Z. Anorg. Chem., 57, 60 (1908).

${ }^{3}$ Swartz, C. E., and Phillips, A. J., Trans. Amer. Inst. Mech. Eng., 111, 333 (1934).

4 Lihl, F., and Buhl, E., Z. Metal., 46, 787 (1958).

\section{CHEMISTRY}

\section{Spin-orbit Coupling and Intermolecular Energy Transfer}

RECENTLY ${ }^{1,2}$ we reported on the intermolecular energy transfer in chemiluminescent solutions, an excited product of the reaction being a donor and anthracene derivatives being acceptors (activators). The activated chemiluminescence spectra were identical with the fluorescence spectra of the activators, indicating that the singlet state of the activator is the excited on $\theta^{2}$. A dependence of the chemiluminescence intensity on the activator concentration was used for estimating the ratio $k_{P A} / f_{P}\left(k_{P A}\right.$, transfer constant, $f_{P}$, emission probability of the excited product, $P^{\prime}$ (Fig. 1)). The relative values of the $k_{P A}$ 's were found. to be greater the more halogens were introduced in the activator molecule and the greater was the atomic weight of the substituent: in this communication those features of the phenomenon are regarded as evidence for the spinorbit coupling in the activator molecule.

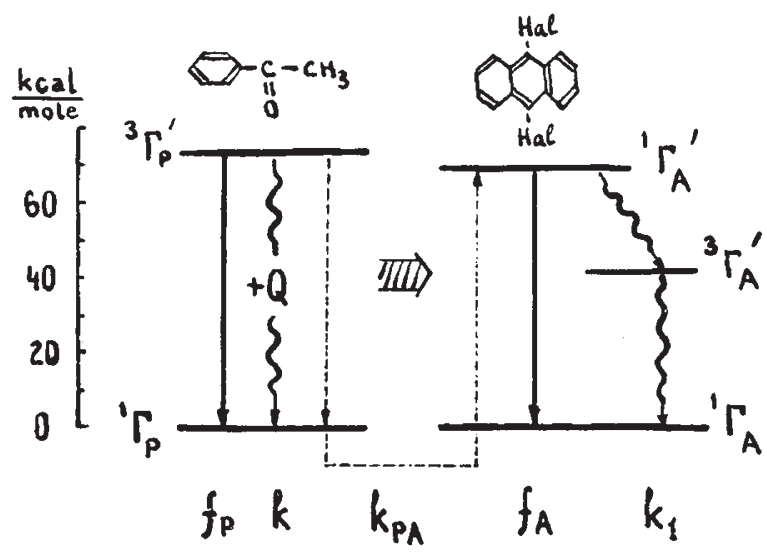

Fig. 1. Energy-levels diagram for the chemiluminescence in ethylbenzene oxidation and for the energy transfer on the anthracene deriva-
tives. The solid, waved and dashed lines represent, respectively, radiative, radiationless transitions and energy transfer. Deactivation by a quencher, $Q$, is discussed in ref. 3

The excited product $P^{\prime}$ formed in the hydrocarbon oxidation was identified as a ketone in its triplet state ${ }^{3}$. Therefore, the energy transfer may be represented as a coupled transition $\left.\right|^{8} \Gamma^{\prime} P^{-\rightarrow}{ }^{1} \Gamma_{P}|\rightarrow|{ }^{1} \Gamma_{A^{n}} \rightarrow^{1} \Gamma_{A}^{\prime} \mid$, as shown in Fig. 1. There were reasons for considering it as an exchange-resonance transfer-its features strongly differed ${ }^{2}$ from those required by the theories of the inductive-resonance or radiative transfer, and the maximum value of $k_{P A}$ (for 9,10-di-bromoanthracene) was only 20-30 times less than the number of collisions. So we believe that the 